\title{
Contribution of hard photon emission to the charge asymmetry in elastic lepton- and antilepton-proton scattering
}

\author{
Andrei Afanasev $\odot^{*}$ \\ Department of Physics, The George Washington University, Washington, D.C. 20052, USA \\ Alexander Ilyichev ${ }^{\dagger}$ \\ Belarusian State University, 220030 Minsk, Belarus \\ and Institute for Nuclear Problems, Belarusian State University, 220006 Minsk, Belarus
}

(Received 9 February 2021; accepted 9 December 2021; published 21 January 2022)

\begin{abstract}
The influence of the hard photon emission on the charge asymmetry in the lepton- and antilepton-proton elastic scattering was evaluated for the first time beyond the ultrarelativistic limit, while retaining the lepton mass at all steps of the calculation. This contribution — responsible for the charge asymmetry—is induced by interference between real photon emission from the lepton and proton. During the calculation any excited states of the intermediated proton were not considered, allowing us to use the standard fermionic propagator for this particle. The infrared divergence extracted using Lorenz-invariant approach of Bardin-Shumeiko is canceled by the corresponding soft part of the two-photon exchange contribution. Numerical analysis was performed within kinematic conditions of Jefferson Lab measurements and MUSE experiment in PSI.
\end{abstract}

DOI: 10.1103/PhysRevD.105.L011301

\section{INTRODUCTION}

The elastic form factors of the proton play an essential role in our understanding the nucleon electromagnetic structure. However $Q^{2}$-dependence of the ratio of the electric to magnetic proton form factors, $G_{E}\left(Q^{2}\right) / G_{M}\left(Q^{2}\right)$, obtained from the unpolarized $[1,2]$ and polarized $[3,4]$ electron elastic scattering data disagreed with each other, and the disagreement was attributed to two-photon exchange effects. The challenge in computing two-photon exchange contribution is in the need for modeling nucleon's structure. It motivated a dedicated program of both direct and indirect experimental measurements of two-photon effects in electron scattering, along with a significant theory effort; the recent status of the problem is reviewed in, e.g., Ref. [5]. Moreover, two-photon effects may have an impact on determination of proton's radius and resolution of the "proton radius puzzle" [6]. Direct measurements of the two-photon effect on the unpolarized scattering cross section [7-10] are based on the observation that this contribution changes its sign with a sign of the scattering lepton, therefore it can be evaluated via measurements of the charge asymmetry. However, the charge asymmetry is caused not only by the two-photon exchange,

\footnotetext{
*afanas@gwu.edu

†ily@hep.by
}

Published by the American Physical Society under the terms of the Creative Commons Attribution 4.0 International license. Further distribution of this work must maintain attribution to the author(s) and the published article's title, journal citation, and DOI. Funded by SCOAP ${ }^{3}$. but also- as required to cancel the infrared divergence- by interference of real photon emission from the lepton and the proton, which is a subject of this paper.

Current research efforts aimed at resolving the abovementioned proton radius puzzle [6] include, in particular, comparison-with a subpercent accuracy-of muon/ antimuon and electron/positron scattering on a proton target in an ongoing MUSE experiment [11] in PSI. In MUSE, the muon momenta are in $100-200 \mathrm{MeV}$ range, of the order of muon's mass, requiring that both the leadingorder expressions for the scattering cross section and QED corrections include muon's mass with no ultrarelativistic approximations usually applied for scattering of electrons.

In many cases for the estimation of the high order QED effects to the exclusive processes the loop corrections (with the additional virtual particle contributions) are calculated exactly or within ultrarelativistic approximation (with respect to lepton's mass) while the real photon emission is considered within the soft photon approximation. For example, in the papers [12] and [13] for Möller and virtual Compton scattering processes, respectively, the virtual QED corrections have been calculated beyond the ultrarelativistic limit but only the soft part of the real photon emission was taken into account.

The charge asymmetry in the leading order of QED in the soft photon approximation was calculated in Ref. [14], while treating the lepton mass exactly. In the present paper we go beyond the soft photon approximation and include, for the first time, the effects of hard photon emission and its influence on the charge asymmetry in elastic lepton- and antilepton- proton scattering. We study effects of hard photon 


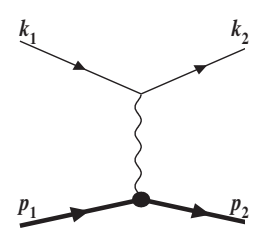

(a)

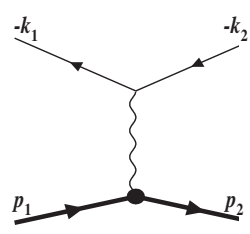

(b)
FIG. 1. Feynman graphs for the lowest-order contribution to elastic $l^{-} p$ (a) and $l^{+} p$ (b) scattering.

emission in various kinematic conditions of experiments targeting the charge asymmetry: MeV energies of MUSE [11] and GeV energies of JLab [7,8]. The infrared divergence is canceled with the corresponding soft part from the twophoton exchange using Lorentz-invariant approach of BardinShumeiko [15]. All calculations were performed without using an ultrarelativistic limit, making them applicable for both low and high energies of scattering leptons.

\section{METHOD OF CALCULATION}

During the calculation we assume that there is no excited states of the intermediated proton-photon vertices. As a result, the proton propagator has a standard for a pointlike fermion. The second assumption is that the on-shell protonphoton vertex,

$$
\Gamma_{\mu}(q)=\gamma_{\mu} F_{d}\left(-q^{2}\right)+\frac{i \sigma_{\mu \nu} q^{\nu}}{2 M} F_{p}\left(-q^{2}\right)
$$

is applicable for the off-shell region. Here $\sigma_{\mu \nu}=i\left[\gamma_{\mu}, \gamma_{\nu}\right] / 2$, $M$ is a proton mass and $q$ is a four-momentum of the virtual photon. The Dirac and Pauli form factors can be expressed through the electric and magnetic ones:

$$
\begin{aligned}
& F_{d}\left(-q^{2}\right)=\frac{G_{E}\left(-q^{2}\right)+\tau G_{M}\left(-q^{2}\right)}{1+\tau}, \\
& F_{p}\left(-q^{2}\right)=\frac{G_{M}\left(-q^{2}\right)-G_{E}\left(-q^{2}\right)}{1+\tau},
\end{aligned}
$$

where $\tau=-q^{2} / 4 M^{2}$.

The lowest-order (Born) contribution to the elastic $l^{\mp} p$ scattering is presented by Feynman graphs in Fig. 1 and it can be described by the following matrix elements:

$$
\begin{aligned}
& \mathcal{M}_{b}^{-}=\frac{i e^{2}}{Q^{2}} \bar{u}\left(k_{2}\right) \gamma^{\mu} u\left(k_{1}\right) \bar{U}\left(p_{2}\right) \Gamma_{\mu}(q) U\left(p_{1}\right), \\
& \mathcal{M}_{b}^{+}=\frac{i e^{2}}{Q^{2}} \bar{u}\left(-k_{1}\right) \gamma^{\mu} u\left(-k_{2}\right) \bar{U}\left(p_{2}\right) \Gamma_{\mu}(q) U\left(p_{1}\right),
\end{aligned}
$$

where $Q^{2}=-q^{2}=-\left(k_{1}-k_{2}\right)^{2}$ and $e=\sqrt{4 \pi \alpha}$. Since the squares of these two matrix elements are identical and are insensitive to the sign of lepton's charge, it is not possible to distinguish the lepton-proton from antilepton-proton scattering processes at the one-photon exchange level. Their contribution to the cross section can be written as

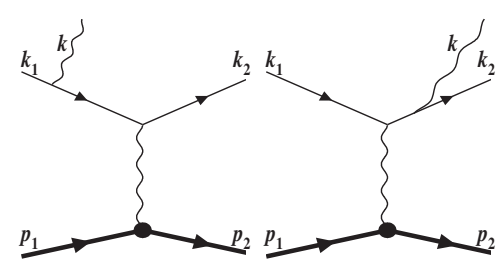

(a)

(b)

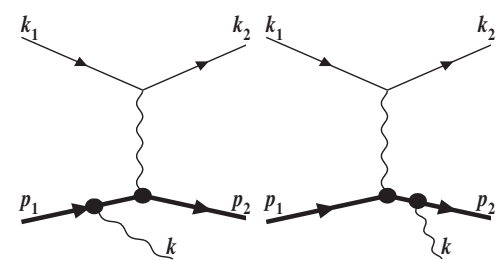

(c)

(d)

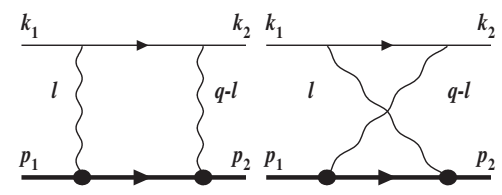

(e)

(f)

FIG. 2. Feynman graphs for the real photon emission from the lepton (a,b) and proton (c,d) legs as well as the direct (e) and cross (f) two-photon exchange within $l^{-} p$-scattering. The similar graphs for $l^{+} p$ scattering processes have an opposite direction for the leptonic arrows and a negative sign for its momenta.

$$
d \sigma_{B}=\frac{1}{2 \sqrt{\lambda_{S}}}\left|\mathcal{M}_{b}^{\mp}\right|^{2} d \Gamma_{2},
$$

where $\lambda_{S}=S^{2}-4 m^{2} M^{2}, S=2 p_{1} k_{1}, m$ is a lepton mass. Following $[16,17]$ we will consider the elastic cross section as a function of $Q^{2}$. As a result the phase space reads:

$$
\begin{aligned}
d \Gamma_{2} & =(2 \pi)^{4} \delta^{4}\left(p_{1}+k_{1}-p_{2}-k_{2}\right) \frac{d^{3} k_{2}}{(2 \pi)^{3} 2 k_{20}} \frac{d^{3} p_{2}}{(2 \pi)^{3} 2 p_{20}} \\
& =\frac{d Q^{2}}{8 \pi \sqrt{\lambda_{S}}} .
\end{aligned}
$$

The Feynman graphs with the real photon emission both from the lepton and proton legs are shown in Figs. 2(a)-2(d) for $l^{-} p$ scattering. The matrix elements corresponding to these processes, as well as the real photon emission in $l^{+} p$ scattering, read:

$$
\begin{aligned}
& \mathcal{M}_{l R}^{-}=-\frac{i e^{3}}{t} \bar{u}\left(k_{2}\right) \varepsilon_{\alpha} \Gamma_{l R}^{\mu \alpha} u\left(k_{1}\right) \bar{U}\left(p_{2}\right) \Gamma_{\mu}(q-k) U\left(p_{1}\right), \\
& \mathcal{M}_{l R}^{+}=\frac{i e^{3}}{t} \bar{u}\left(-k_{1}\right) \varepsilon_{\alpha} \bar{\Gamma}_{l R}^{\mu \alpha} u\left(-k_{2}\right) \bar{U}\left(p_{2}\right) \Gamma_{\mu}(q-k) U\left(p_{1}\right), \\
& \mathcal{M}_{h R}^{-}=\frac{i e^{3}}{Q^{2}} \bar{u}\left(k_{2}\right) \gamma_{\mu} u\left(k_{1}\right) \bar{U}\left(p_{2}\right) \varepsilon_{\alpha} \Gamma_{h R}^{\mu \alpha} U\left(p_{1}\right), \\
& \mathcal{M}_{h R}^{+}=\frac{i e^{3}}{Q^{2}} \bar{u}\left(-k_{1}\right) \gamma_{\mu} u\left(-k_{2}\right) \bar{U}\left(p_{2}\right) \varepsilon_{\alpha} \Gamma_{h R}^{\mu \alpha} U\left(p_{1}\right),
\end{aligned}
$$

where $t=-(q-k)^{2}=-\left(p_{2}-p_{1}\right)^{2}, \quad \varepsilon_{\alpha}$ is the photon polarized vector and 


$$
\begin{aligned}
\Gamma_{l R}^{\mu \alpha}= & \left(\frac{k_{1 \alpha}}{k k_{1}}-\frac{k_{2 \alpha}}{k k_{2}}\right) \gamma^{\mu}-\frac{\gamma^{\mu} \hat{k} \gamma^{\alpha}}{2 k_{1} k}-\frac{\gamma^{\alpha} \hat{k} \gamma^{\mu}}{2 k_{2} k}, \\
\bar{\Gamma}_{l R}^{\mu \alpha}= & \left(\frac{k_{1 \alpha}}{k k_{1}}-\frac{k_{2 \alpha}}{k k_{2}}\right) \gamma^{\mu}-\frac{\gamma^{\alpha} \hat{k} \gamma^{\mu}}{2 k_{1} k}-\frac{\gamma^{\mu} \hat{k} \gamma^{\alpha}}{2 k_{2} k}, \\
\Gamma_{h R}^{\mu \alpha}= & \Gamma^{\mu}(q) \frac{\hat{p}_{1}-\hat{k}+M}{2 p_{1} k} \Gamma^{\alpha}(-k) \\
& -\Gamma^{\alpha}(-k) \frac{\hat{p}_{2}+\hat{k}+M}{2 p_{2} k} \Gamma^{\mu}(q) .
\end{aligned}
$$

The part of the cross section with the interference between the real photon emissions from hadron and lepton lines reads:

$$
d \sigma_{R}^{\mp}=\frac{1}{2 \sqrt{\lambda_{S}}}\left(\mathcal{M}_{l R}^{\mp} \mathcal{M}_{h R}^{\mp \dagger}+\mathcal{M}_{h R}^{\mp} \mathcal{M}_{l R}^{\mp \dagger}\right) d \Gamma_{3},
$$

where, according to the definitions of Refs. $[16,17]$, the phase space can be presented as

$$
\begin{aligned}
d \Gamma_{3}= & (2 \pi)^{4} \delta^{4}\left(p_{1}+k_{1}-p_{2}-k_{2}-k\right) \\
& \times \frac{d^{3} k}{(2 \pi)^{3} 2 k_{0}} \frac{d^{3} k_{2}}{(2 \pi)^{3} 2 k_{20}} \frac{d^{3} p_{2}}{(2 \pi)^{3} 2 p_{20}} \\
= & \frac{d Q^{2} d v d t d \phi_{k}}{2^{8} \pi^{4} \sqrt{\lambda_{S} Q^{2}\left(Q^{2}+4 M^{2}\right)}} .
\end{aligned}
$$

Here $v=\left(p_{1}+q\right)^{2}-M^{2}$ is a photonic variable defining inelasticity, $\phi_{k}$ is an angle between $\left(\mathbf{k}_{1}, \mathbf{k}_{2}\right)$ and $(\mathbf{k}, \mathbf{q})$ planes in the rest frame $\left(\mathbf{p}_{1}=0\right)$. It should be noted that for the radiative process the energy of the scattering lepton reads:

$$
E^{\prime}=\frac{S-Q^{2}-v}{2 M} .
$$

The upper limit of integration with respect to the variable $v$ is defined as

$v_{m}=\frac{1}{2 m^{2}}\left(\sqrt{\lambda_{S}} \sqrt{Q^{2}\left(Q^{2}+4 m^{2}\right)}-2 m^{2} Q^{2}-Q^{2} S\right)$.

In experiment, however, the influence of the hard real photon emission to the asymmetry can be essentially reduced by applying a kinematic cut $v_{\text {cut }}$ on the inelasticity which is a measured quantity in the elastic lepton-proton scattering.

Performing the direct calculation, one can find that the interference terms for $l^{-} p$ and $l^{+} p$ have opposite signs,

$$
d \sigma_{R}^{+}=-d \sigma_{R}^{-}
$$

In order to estimate this contribution to the elastic process, it is necessary to integrate $d \sigma_{R}^{\mp}$ over three photonic variables: $v, t$ and $\phi_{k}$. However, since the expressions (8) contain the infrared divergent terms at $k \rightarrow 0$, it is not possible to perform the integration in the straightforward way. Following to the Bardin-Shumeiko approach [15] for the extraction of the infrared (IR) divergence, the identity transformation has to be performed:

$$
d \sigma_{R}^{\mp}=d \sigma_{R}^{\mp}-d \sigma_{\mathrm{IR}}^{\mp}+d \sigma_{\mathrm{IR}}^{\mp}=d \sigma_{F}^{\mp}+d \sigma_{\mathrm{IR}}^{\mp} .
$$

In the infrared-free term $d \sigma_{F}^{ \pm}$the integration can be performed over three photonic variables without any restrictions. The infrared term $d \sigma_{\mathrm{IR}}^{ \pm}$can be obtained by the substitution to the expressions (6) the soft parts of the vertexes with a real photon emission (7) that survive when $k \rightarrow 0$ :

$$
\begin{aligned}
\Gamma_{l R \text { soft }}^{\mu \alpha} & =\left(\frac{k_{1 \alpha}}{k k_{1}}-\frac{k_{2 \alpha}}{k k_{2}}\right) \gamma^{\mu}, \\
\bar{\Gamma}_{l R \text { soft }}^{\mu \alpha} & =\left(\frac{k_{1 \alpha}}{k k_{1}}-\frac{k_{2 \alpha}}{k k_{2}}\right) \gamma^{\mu}, \\
\Gamma_{h R \text { soft }}^{\mu \alpha} & =\left(\frac{p_{1 \alpha}}{k p_{1}}-\frac{p_{2 \alpha}}{k p_{2}}\right) \Gamma^{\mu}(q) .
\end{aligned}
$$

As a result, $d \sigma_{\mathrm{IR}}^{ \pm}$is factorized in front of the Born contribution the following way:

$$
\frac{d \sigma_{\mathrm{IR}}^{\mp}}{d Q^{2}}=\mp \frac{\alpha}{\pi}\left(\delta_{S}+\delta_{H}\right) \frac{d \sigma_{B}}{d Q^{2}} .
$$

The quantities $\delta_{S}$ and $\delta_{H}$ appear after splitting the integration region over the inelasticity $v$ by introducing the infinitesimal parameter $\bar{v}$

$$
\begin{gathered}
\delta_{S}=\frac{1}{\pi} \int_{0}^{\bar{v}} d v \int \frac{d^{3} k}{k_{0}} F_{\mathrm{IR}} \delta\left(\left(p_{1}+q-k\right)^{2}-M^{2}\right), \\
\delta_{H}=\frac{1}{\pi} \int_{\bar{v}}^{v_{\text {cut }}} d v \int \frac{d^{3} k}{k_{0}} F_{\mathrm{IR}} \delta\left(\left(p_{1}+q-k\right)^{2}-M^{2}\right),
\end{gathered}
$$

where

$$
F_{\text {IR }}=-\frac{1}{2}\left(\frac{k_{1}}{k_{1} k}-\frac{k_{2}}{k_{2} k}\right)\left(\frac{p_{1}}{p_{1} k}-\frac{p_{2}}{p_{2} k}\right) .
$$

Performing the integration in $\delta_{S}$ (using the photon mass $\lambda$ for the infrared divergence regularization) and $\delta_{H}$, we can find that

$$
\begin{gathered}
\delta_{S}=\delta_{S}^{1}-2\left(S L_{S}-X L_{X}\right) \log \left[\frac{\bar{v}}{M \lambda}\right], \\
\delta_{H}=\delta_{H}^{1}-2\left(S L_{S}-X L_{X}\right) \log \left[\frac{v_{\text {cut }}}{\bar{v}}\right],
\end{gathered}
$$

where

$$
\begin{aligned}
& L_{S}=\frac{1}{\sqrt{\lambda_{S}}} \log \frac{S+\sqrt{\lambda_{S}}}{S-\sqrt{\lambda_{S}}}, \\
& L_{X}=\frac{1}{\sqrt{\lambda_{X}}} \log \frac{X+\sqrt{\lambda_{X}}}{X-\sqrt{\lambda_{X}}},
\end{aligned}
$$

$X=S-Q^{2}$ and $\lambda_{X}=X^{2}-4 M^{2} m^{2}$. The quantities $\delta_{S}^{1}$ and $\delta_{H}^{1}$ have a rather complicated structure and depend neither on $\bar{v}$ nor on $\lambda$. Some specific detail of calculations, as well as the explicit expressions for $\delta_{S}^{1}$ and $\delta_{H}^{1}$, can be found in [15]. Notice that both of these quantities vanish when $Q^{2}=0$. 
As a result, the sum of $\delta_{S}$ and $\delta_{H}$ is free of the separation parameter $\bar{v}$ but it contains dependence on the fictitious photon mass $\lambda$. Such dependence means that $\delta_{S}$ (as well as the sum of $\delta_{S}$ and $\delta_{H}$ ) contains the infrared divergence that should be canceled by adding the two-photon exchange contribution. However, as we can see below, the latter has a rather complicated structure due to the integration over the virtual 4-momentum. Therefore in order to remove the dependence on $\lambda$ analytically it is necessary to extract the infrared divergence from the two-photon exchange contribution into a separate term.

As shown in Figs. 2(e) and 2(f), the matrix elements with two-photon exchange contribution to the elastic $l^{\mp} p$ scattering can be separated into the direct $\mathcal{M}_{d 2 \gamma}^{\mp}$ and cross $\mathcal{M}_{x 2 \gamma}^{\mp}$ terms that can be presented as loop integrals in a following way:

$$
\begin{aligned}
& \mathcal{M}_{d 2 \gamma}^{-}=\frac{e^{4}}{(2 \pi)^{4}} \int \frac{d^{4} l}{l^{2}(l-q)^{2}} \bar{u}\left(k_{2}\right) \gamma^{\nu} \frac{\hat{k}_{1}-\hat{l}+m}{l^{2}-2 k_{1} l} \gamma^{\mu} u\left(k_{1}\right) \bar{U}\left(p_{2}\right) \Gamma_{\nu}(q-l) \frac{\hat{p}_{1}+\hat{l}+M}{l^{2}+2 p_{1} l} \Gamma_{\mu}(l) U\left(p_{1}\right), \\
& \mathcal{M}_{d 2 \gamma}^{+}=\frac{e^{4}}{(2 \pi)^{4}} \int \frac{d^{4} l}{l^{2}(l-q)^{2}} \bar{u}\left(-k_{1}\right) \gamma^{\mu} \frac{\hat{l}-\hat{k}_{1}+m}{l^{2}-2 k_{1} l} \gamma^{\nu} u\left(-k_{2}\right) \bar{U}\left(p_{2}\right) \Gamma_{\nu}(q-l) \frac{\hat{p}_{1}+\hat{l}+M}{l^{2}+2 p_{1} l} \Gamma_{\mu}(l) U\left(p_{1}\right), \\
& \mathcal{M}_{x 2 \gamma}^{-}=\frac{e^{4}}{(2 \pi)^{4}} \int \frac{d^{4} l}{l^{2}(l-q)^{2}} \bar{u}\left(k_{2}\right) \gamma^{\nu} \frac{\hat{k}_{1}-\hat{l}+m}{l^{2}-2 k_{1} l} \gamma^{\mu} u\left(k_{1}\right) \bar{U}\left(p_{2}\right) \Gamma_{\mu}(l) \frac{\hat{p}_{2}-\hat{l}+M}{l^{2}-2 p_{2} l} \Gamma_{\nu}(q-l) U\left(p_{1}\right), \\
& \mathcal{M}_{x 2 \gamma}^{+}=\frac{e^{4}}{(2 \pi)^{4}} \int \frac{d^{4} l}{l^{2}(l-q)^{2}} \bar{u}\left(-k_{1}\right) \gamma^{\mu} \frac{\hat{l}-\hat{k}_{1}+m}{l^{2}-2 k_{1} l} \gamma^{\nu} u\left(-k_{2}\right) \bar{U}\left(p_{2}\right) \Gamma_{\mu}(l) \frac{\hat{p}_{2}-\hat{l}+M}{l^{2}-2 p_{2} l} \Gamma_{\nu}(q-l) U\left(p_{1}\right) .
\end{aligned}
$$

Note that all of these matrix elements contain the infrared divergence at $l=0$ and $l=q$ points.

The lowest-order two-photon exchange contribution to the elastic $l^{\mp} p$ cross section has a form

$$
\begin{aligned}
d \sigma_{2 \gamma}^{\mp}= & \frac{1}{2 \sqrt{\lambda_{S}}}\left[\mathcal{M}_{b}^{\mp}\left(\mathcal{M}_{d 2 \gamma}^{\mp}+\mathcal{M}_{x 2 \gamma}^{\mp}\right)^{\dagger}\right. \\
& \left.+\left(\mathcal{M}_{d 2 \gamma}^{\mp}+\mathcal{M}_{x 2 \gamma}^{\mp}\right) \mathcal{M}_{b}^{\mp \dagger}\right] d \Gamma_{2} .
\end{aligned}
$$

Once again we can find that

$$
d \sigma_{2 \gamma}^{+}=-d \sigma_{2 \gamma}^{-} .
$$

The required term with the infrared divergence is extracted from two-photon exchange contribution according to the prescription presented in [14] and it has a form

$$
\frac{d \sigma_{2 \gamma \mathrm{IR}}^{\mp}}{d Q^{2}}=\mp \frac{\alpha}{\pi}\left(\delta_{2 \gamma}^{1}\left(Q^{2}\right)+\left(S L_{S}-X L_{X}\right) \log \left[\frac{Q^{2}}{\lambda^{2}}\right]\right) \frac{d \sigma_{B}}{d Q^{2}},
$$

where the quantity $\delta_{2 \gamma}^{1}\left(Q^{2}\right)$ has a rather complicated structure and does not depend on the fictitious photon mass $\lambda$. Its explicit expression can be found in [14].

During the calculation of radiative corrections to the leptonic current (see, e.g., [16,17]) the ultraviolet divergences from the additional virtual particle contributions such as the vertex and lepton self-energies are removed by using an on-shell renormalization scheme: the considered ultraviolet-free contributions are obtained by subtraction of their value at $Q^{2}=0$. As a result, these contributions vanish at $Q^{2} \rightarrow 0$. Similar behavior we can observed for the charge asymmetry (see, e.g., [18]). However, as opposed to $\delta_{S}$ and $\delta_{H}$, direct calculations give $\delta_{2 \gamma}^{1}(0) \neq 0$. Since the two-photon exchange process also belongs to radiative corrections but without ultraviolet divergence, in order to fix this disagreement, an on-shell renormalization procedure can be applied. As a result, the corrected contribution reads:

$\frac{d \hat{\sigma}_{2 \gamma \mathrm{IR}}^{\mp}}{d Q^{2}}=\mp \frac{\alpha}{\pi}\left(\hat{\delta}_{2 \gamma}^{1}+\left(S L_{S}-X L_{X}\right) \log \left[\frac{Q^{2}}{\lambda^{2}}\right]\right) \frac{d \sigma_{B}}{d Q^{2}}$,

where $\hat{\delta}_{2 \gamma}^{1}=\delta_{2 \gamma}^{1}\left(Q^{2}\right)-\delta_{2 \gamma}^{1}(0)$. Notice that in the ultrarelativistic approximation the obtained expression exactly matches Eq. (2.20) from the review [5].

The sum of Eq. (15) with Eq. (24)

$$
\frac{d \sigma_{\mathrm{IR}}^{\mp}}{d Q^{2}}+\frac{d \hat{\sigma}_{2 \gamma \mathrm{IR}}^{\mp}}{d Q^{2}}=\mp \frac{\alpha}{\pi} \delta_{V R} \frac{d \sigma_{B}}{d Q^{2}}
$$

is infrared-free since

$$
\delta_{V R}=\left(\left(S L_{S}-X L_{X}\right) \log \left[\frac{Q^{2} M^{2}}{v_{\mathrm{cut}}^{2}}\right]+\delta_{S}^{1}+\delta_{H}^{1}+\hat{\delta}_{2 \gamma}^{1}\right)
$$

does not depend on $\lambda$, either.

Finally, the lowest order of the charge-odd contribution to the elastic lepton-proton cross section reads:

$$
\frac{d \sigma_{\text {odd }}^{\mp}}{d Q^{2}}=\frac{d \sigma_{F}^{\mp}}{d Q^{2}} \mp \frac{\alpha}{\pi} \delta_{V R} \frac{d \sigma_{B}}{d Q^{2}} .
$$

Since the hard photon contribution to the charge asymmetry was evaluated for the first time, there is no explicit expression in the literature for $d \sigma_{F}^{\mp} / d Q^{2}$. This quantity has a structure similar to Eq. (43) of [17], i.e., it can be expressed as a three-dimensional integral with respect to $\phi_{k}, \tau$ and $v$ of the difference $d \sigma_{R}-d \sigma_{R}^{\mathrm{IR}}$. 

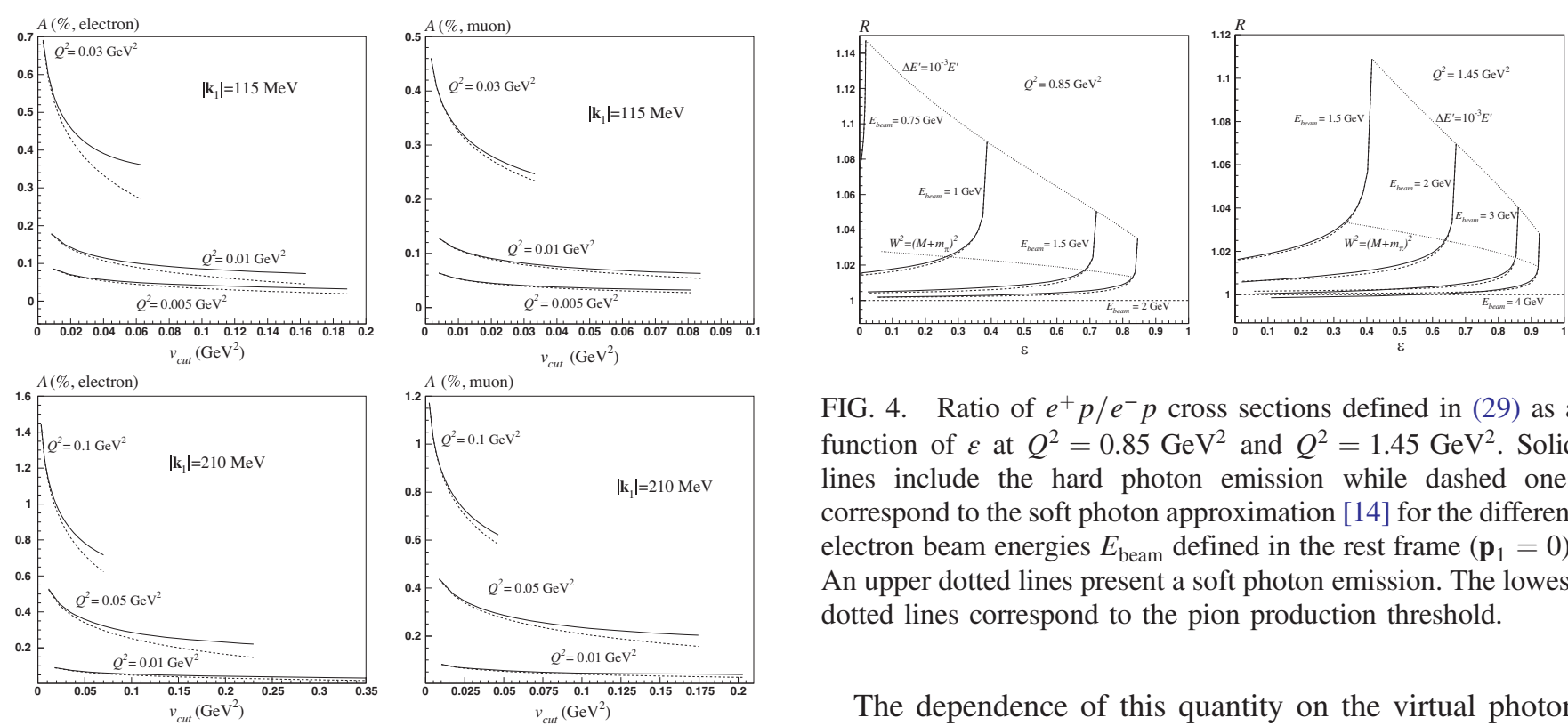

FIG. 3. A charge asymmetry defined in (28) vs the value of the inelasticity cut for elastic $e^{\mp} p$ and $\mu^{\mp} p$ scattering with the beam momenta $115 \mathrm{MeV}$ and $210 \mathrm{MeV}$ defined in the rest frame $\left(\mathbf{p}_{1}=0\right)$. The dashed lines correspond to the soft photon approximation [14]. The solid lines include the hard photon emission.

\section{NUMERICAL RESULTS}

Based on the obtained expressions for the numerical estimation of radiative effects, a new version of the FORTRAN code MASCARAD [16] is being developed. The parametrization of proton form factors were taken from Kelly's paper [19].

The dependence of the charge asymmetry

$$
A=\frac{d \sigma_{\text {odd }}^{+} / d Q^{2}-d \sigma_{\text {odd }}^{-} / d Q^{2}}{d \sigma_{B} / d Q^{2}},
$$

on the value of the upper integration limit over the inelasticity $v_{\text {cut }}$ for different lepton beams and $Q^{2}$ is presented in Fig. 3 under MUSE kinematic conditions [11]. The dashed lines correspond to the contribution of only the soft photon approximation calculated in [14], while the solid lines include the hard photon emission presented in this paper. From these plots one can see that at the fixed lepton momentum the asymmetry decreases more rapidly with growing $v_{\text {cut }}$ without hard photon contribution. It can be also seen that the magnitude of the asymmetry is reduced with increasing $Q^{2}$. Moreover, the value of this asymmetry is higher for the lighter lepton.

Another important quantity is the ratio of $e^{+} p / e^{-} p$ cross sections that can be defined as

$$
R=\frac{d \sigma_{B} / d Q^{2}+d \sigma_{\text {odd }}^{+} / d Q^{2}}{d \sigma_{B} / d Q^{2}+d \sigma_{\text {odd }}^{-} / d Q^{2}}
$$

FIG. 4. Ratio of $e^{+} p / e^{-} p$ cross sections defined in (29) as a function of $\varepsilon$ at $Q^{2}=0.85 \mathrm{GeV}^{2}$ and $Q^{2}=1.45 \mathrm{GeV}^{2}$. Solid lines include the hard photon emission while dashed ones correspond to the soft photon approximation [14] for the different electron beam energies $E_{\text {beam }}$ defined in the rest frame $\left(\mathbf{p}_{1}=0\right)$. An upper dotted lines present a soft photon emission. The lowest dotted lines correspond to the pion production threshold.

The dependence of this quantity on the virtual photon polarization $\varepsilon$ at JLab kinematic conditions is shown in Fig. 4. Since, according to [20], $\varepsilon$ beyond the ultrarelativistic approximation has a form

$$
\varepsilon=\left(1+2(1+\tau) \frac{\left(Q^{2}-2 m^{2}\right)}{4 E_{\text {beam }} E^{\prime}-Q^{2}}\right)^{-1}
$$

and for the bremsstrahlung process $E^{\prime}$ depends on $v$ as defined in Eq. (10), it is necessary to integrate this effect over $v$ (from 0 to $v_{\text {cut }}$ ) to estimate its contribution to the elastic process. As a result for the fixed electron beam energies (solid lines) the small $\varepsilon$ corresponds to the hard photon contribution in the region where the ratio is closer to unity. Analogously to the previous plots, the soft photon approximation is presented by dashed lines, while the solid lines contain the hard photon emission. Similar to the experimental observation $[7,8]$, the cross section ratio with the soft photon emission (upper dotted lines) decreases with growing $\varepsilon$. The lowest dotted lines correspond the pion threshold when the quantity $W^{2}=\left(p_{1}+q\right)^{2}$ reaches a value $\left(M+m_{\pi}\right)^{2}$ and, together with the soft photon, one undetected pion could to be produced.

\section{CONCLUSION}

The contribution of the hard photon emission to the charge asymmetry in lepton-proton scattering was estimated for the first time beyond the ultrarelativistic limit, while retaining lepton mass during the entire calculation.

Only two assumptions were used in the calculation: (I) We did not consider inelastic excitations of the intermediate proton and used a standard fermionic propagator for it; (II) The on-shell proton vertex with the Dirac and Pauli form factors were used in the off-shell region.

The numerical results shown that if the final lepton momentum is fixed, the asymmetry decreases with growing 
energy of the unobserved photon. This asymmetry is sensitive to the lepton mass: its value is higher for the lighter lepton.

The next planned steps consist in the update of FORTRAN code MASCARAD on the calculation of radiative corrections from the lepton current similarly to [17]. It will also be interesting to consider in the future the contribution of baryonic ( $\Delta$ etc.) intermediate states since it is not obvious that their contribution is negligible. In addition, we will implement the obtained results into a Monte-Carlo generator ELRADGEN [21,22] for simulation of hard photon emission in future experiments.
[1] L. Andivahis, P. E. Bosted, A. Lung, L. M. Stuart, J. Alster, R. G. Arnold et al., Phys. Rev. D 50, 5491 (1994).

[2] I. A. Qattan et al., Phys. Rev. Lett. 94, 142301 (2005).

[3] M. Jones et al. (Jefferson Lab Hall A Collaboration), Phys. Rev. Lett. 84, 1398 (2000).

[4] O. Gayou et al. (Jefferson Lab Hall A Collaboration), Phys. Rev. Lett. 88, 092301 (2002).

[5] A. Afanasev, P. Blunden, D. Hasell, and B. Raue, Prog. Part. Nucl. Phys. 95, 245 (2017).

[6] R. Pohl et al., Nature (London) 466, 213 (2010).

[7] D. Adikaram et al. (CLAS Collaboration), Phys. Rev. Lett. 114, 062003 (2015).

[8] D. Rimal et al. (CLAS Collaboration), Phys. Rev. C 95, 065201 (2017).

[9] I. A. Rachek, J. Arrington, V. F. Dmitriev, V. V. Gauzshtein, R. E. Gerasimov, A. V. Gramolin et al., Phys. Rev. Lett. 114, 062005 (2015).

[10] B. S. Henderson et al. (OLYMPUS Collaboration), Phys. Rev. Lett. 118, 092501 (2017).

[11] R. Gilman et al., Technical Design Report for the Paul Scherrer Institute Experiment R-12-01.1: Studying the Proton "Radius" Puzzle with $\mu$ p Elastic Scattering (2017), arXiv:1709.09753.
[12] N. Kaiser, J. Phys. G 37, 115005 (2010).

[13] M. Vanderhaeghen, J. M. Friedrich, D. Lhuillier, D. Marchand, L. Van Hoorebeke, and J. Van de Wiele, Phys. Rev. C 62, 025501 (2000).

[14] O. Koshchii and A. Afanasev, Phys. Rev. D 96, 016005 (2017).

[15] D. Bardin and N. Shumeiko, Nucl. Phys. B127, 242 (1977).

[16] A. Afanasev, I. Akushevich, and N. Merenkov, Phys. Rev. D 64, 113009 (2001).

[17] I. Akushevich, H. Gao, A. Ilyichev, and M. Meziane, Eur. Phys. J. A 51, 1 (2015).

[18] B. S. Henderson et al. (OLYMPUS Collaboration), Phys. Rev. Lett. 118, 092501 (2017).

[19] J. J. Kelly, Phys. Rev. C 70, 068202 (2004).

[20] B. M. Preedom and R. Tegen, Phys. Rev. C 36, 2466 (1987).

[21] A. V. Afanasev, I. Akushevich, A. Ilyichev, and B. Niczyporuk, Czech. J. Phys. 53, B449 (2003), https:// arxiv.org/abs/hep-ph/0308106.

[22] I. Akushevich, O. Filoti, A. Ilyichev, and N. Shumeiko, Comput. Phys. Commun. 183, 1448 (2012). 